\title{
The relation between the Toda hierarchy and the KdV hierarchy
}

\author{
Yunbo Zeng Runliang Lin Xin Cao \\ Department of Applied Mathematics, Tsinghua University, Beijing 100084, China.
}

\begin{abstract}
Under three relations connecting the field variables of Toda flows and that of $\mathrm{KdV}$ flows, we present three new sequences of combination of the equations in the Toda hierarchy which have the KdV hierarchy as a continuous limit. The relation between the Poisson structures of the $\mathrm{KdV}$ hierarchy and the Toda hierarchy in continuous limit is also studied.
\end{abstract}

PACS: $02.90+\mathrm{p} ; 03.20+\mathrm{i}$

Keywords: Continuous limit; Recombination equation; Toda hierarchy; KdV hierarchy; Poisson structure.

It is well known that the KdV-type equations can be obtained as continuous limits of suitably chosen discrete integrable systems. The relation between KdV equation and continuous limit of Toda lattice was first described by Toda and Wadati囵. The limit process in the realm of inverse scattering was discussed in [2]. Kupershmidt 3] presented a general setting for the integrable discrete system and studied the limit case in this framework.

In recent years the discussion of relation between a hierarchy of soliton equations and continuous limit of a hierarchy of integrable discrete systems has attracted some attention ${ }^{[4-9]}$, since the relation provides method of deforming the soliton hierarchy. One of the authors and RauchWojciechowski 4 first proposed a recombination method to study the relation between a hierarchy of KdV flows and continuous limit of a hierarchy of Kac-Van Moerbeke flows. This recombination method was also studied in [6,7]. An asymptotic series (in the lattice spacing) for field variables of Toda hierarchy in terms of field variable of $\mathrm{KdV}$ hierarchy was proposed in [5] in order that the series be approximate solution to the Toda flows to high accuracy and by choosing the initial data of the Toda flows in a canonical way the behavior of a certain Toda flow can mimic the $\mathrm{KdV}$ flows. Then a conjecture on the relation between KdV hierarchy and limit of Toda hierarchy was suggested. The main purpose of this letter is to present clear and conclusive statement 
for the relation between $\mathrm{KdV}$ hierarchy and continuous limit of Toda hierarchy by using three relations connecting the field variables of Toda flows and that of $\mathrm{KdV}$ flows proposed in [5]. We construct three new sequences of recombination equations in the Toda hierarchy which have the $\mathrm{KdV}$ hierarchy as continuous limit. Also we present the relation between the Poisson structures of the KdV hierarchy and Toda hierarchy in continuous limit. The method can be applied to all the relations connecting the field variables of Toda flows and that of KdV flows.

First, we briefly describe the Toda hierarchy and the KdV hierarchy as presented in $[10,11]$. Consider the following discrete isospectral problem,

$$
\left(E+w+v E^{(-1)}\right) y=\lambda y
$$

where $w=w(n, t)$ and $v=v(n, t)$ depend on integer $n \in \mathbf{Z}$ and $t \in \mathbf{R}, \lambda$ is the spectral parameter, shift operator $E$ is defined as

$$
(E f)(n)=f(n+1), \quad f^{(k)}(n)=E^{(k)} f(n)=f(n+k), \quad n \in \mathbf{Z} .
$$

The equation in the Toda hierarchy associated with (1) can be written as following Hamiltonian equation 10

$$
\left(\begin{array}{l}
w \\
v
\end{array}\right)_{t_{m}}=J K_{m+1}=J \frac{\delta H_{m+1}}{\delta u}, \quad m=1,2, \cdots,
$$

where $\frac{\delta}{\delta u}=\left(\frac{\delta}{\delta w}, \frac{\delta}{\delta v}\right)^{T}$, Poisson tensor $J$, Hamiltonian $H_{i}$ and $K_{i}$ are defined as

$$
\begin{gathered}
J \equiv\left(\begin{array}{cc}
0 & J_{12} \\
J_{21} & 0
\end{array}\right) \equiv\left(\begin{array}{cc}
0 & (1-E) v \\
v\left(E^{(-1)}-1\right) & 0
\end{array}\right), \\
K_{i}=\frac{\delta H_{i}}{\delta u}=\left(\begin{array}{c}
-b_{i}^{(1)} \\
\frac{a_{i}}{v}
\end{array}\right), \quad H_{i}=-\frac{b_{i+1}}{i}, \quad i=0,1, \cdots,
\end{gathered}
$$

with $a_{0}=\frac{1}{2}, b_{0}=0$, and

$$
b_{i+1}^{(1)}=w b_{i}^{(1)}-\left(a_{i}^{(1)}+a_{i}\right), \quad a_{i+1}^{(1)}-a_{i+1}=w\left(a_{i}^{(1)}-a_{i}\right)+v b_{i}-v^{(1)} b_{i}^{(2)}, \quad c_{i}=-v b_{i}^{(1)}, \quad i=0,1, \cdots .
$$

The equations (2) have the bi-Hamiltonian formulation

$$
\begin{gathered}
G K_{i-1}=J K_{i}, \quad i=1,2, \cdots \\
G \equiv\left(\begin{array}{cc}
v E^{(-1)}-v^{(1)} E & w(1-E) v \\
v\left(E^{(-1)}-1\right) w & v\left(E^{(-1)}-E\right) v
\end{array}\right)
\end{gathered}
$$

where $G$ is the second Poisson tensor. We take $a_{1}=0$, then the first four $K_{i}$ 's are

$$
K_{0}=\left(\begin{array}{c}
0 \\
\frac{1}{2 v}
\end{array}\right), \quad K_{1}=\left(\begin{array}{c}
1 \\
0
\end{array}\right), \quad K_{2}=\left(\begin{array}{c}
w \\
1
\end{array}\right), \quad K_{3}=\left(\begin{array}{c}
v+v^{(1)}+w^{2} \\
w+w^{(-1)}
\end{array}\right) .
$$


The Schrödinger spectral problem is of the form

$$
\left(\partial_{x}^{2}+q-\bar{\lambda}\right) \bar{y}=0
$$

which is associated with the following KdV hierarchy 11]

$$
q_{t_{m}}=B_{0} P_{m}=B_{0} \frac{\delta \bar{H}_{m}}{\delta q}, \quad m=1,2, \cdots
$$

where the vector field possesses the bi-Hamiltonian formulation with two Poisson tensors $B_{0}$ and $B_{1}$

$$
\begin{gathered}
B_{0} P_{k+1}=B_{1} P_{k}, \quad k=0,1, \cdots, \\
B_{0}=\partial \equiv \partial_{x}, \quad B_{1}=\frac{1}{4} \partial^{3}+q \partial+\frac{1}{2} q_{x}, \quad \bar{H}_{i}=\frac{4 \bar{b}_{i+2}}{2 i+1}, \quad i=0,1, \cdots,
\end{gathered}
$$

with $\bar{b}_{0}=0, \bar{b}_{1}=1$, and

$$
\bar{b}_{i+1}=\left(\frac{1}{4} \partial^{2}+q-\frac{1}{2} \partial^{-1} q_{x}\right) \bar{b}_{i}, \quad i=0,1, \cdots,
$$

where $\partial^{-1} \partial=\partial \partial^{-1}=1$. The first three $P_{k}$ 's are

$$
P_{0}=2, \quad P_{1}=q, \quad P_{2}=\frac{1}{4}\left(3 q^{2}+q_{x x}\right) .
$$

The well-known KdV equation reads

$$
q_{t_{2}}=\frac{1}{4}\left(3 q^{2}+q_{x x}\right)_{x}
$$

Consider the Toda hierarchy on a lattice with a small step $h$. We interpolate the sequences $(w(n))$ and $(v(n))$ with two smooth functions of a continuous variable $x$, and relate $w(n)$ and $v(n)$ to $q(x)$ and $g(x)$ by

$$
\begin{gathered}
w(n)=-2+\frac{1}{2} q(x) h^{2}+g(x) h^{3}, \quad v(n)=1+\frac{1}{2} q(x) h^{2}-g(x) h^{3}, \\
E^{(k)} w(n)=-2+\frac{1}{2} q(x+k h) h^{2}+g(x+k h) h^{3}, \quad E^{(k)} v(n)=1+\frac{1}{2} q(x+k h) h^{2}-g(x+k h) h^{3},
\end{gathered}
$$

where $g(x)$ is given by (14). Also we define

$$
\lambda=\bar{\lambda} h^{2}, \quad y(n)=\bar{y}(x)
$$

Then it is easy to see that the spectral problem operators in (1) has the expansion

$$
\left(E+w+v E^{(-1)}-\lambda\right) y(n)=h^{2}\left(\partial^{2}+q-\bar{\lambda}\right) \bar{y}(x)+O\left(h^{3}\right)
$$

which implies that the Toda spectral problem goes to the Schrödinger spectral problem in a continuous limit. The expansion (13) doesn't depend on the choice of $g(x)$. Gieseker proposed a 
way in [5] to choose $g(x)$ by requirement that $w(n)$ and $v(n)$ given by (11) be approximate solution to the Toda flows to different accuracy when $q(x)$ satisfies the KdV equation. For example, the first three choices of $g(x)=g_{1}(x)+g_{2}(x) h$ are as follows, respectively

$$
\begin{gathered}
g_{1}(x)=g_{2}(x)=0, \\
g_{1}(x)=\frac{1}{8} q_{x}(x), \quad g_{2}(x)=0 \\
g_{1}(x)=\frac{1}{8} q_{x}(x), \quad g_{2}(x)=-\frac{1}{32} q^{2}(x) h .
\end{gathered}
$$

It was examined in [5] that suitable chosen combination of first $K_{i}$ 's goes to first $P_{i}$ 's in continuous limit. These results suggest that there exists some relation between the KdV hierarchy and continuous limit of Toda hierarchy. We will show that under (11) with (14a), (14b) and (14c), respectively, a certainly defined combinations of the equations in the Toda hierarchy have the $\mathrm{KdV}$ hierarchy as a continuous limit. In order to do so, we need following lemmas.

Lemma 1. Under the definition (11a), we have

$$
K_{i} \equiv\left(\begin{array}{c}
-b_{i}^{(1)} \\
\frac{a_{i}}{v}
\end{array}\right)=\left(\begin{array}{c}
\alpha_{i} \\
\gamma_{i}
\end{array}\right)+O(h), \quad i=0,1, \cdots
$$

where the constants $\alpha_{i}$ and $\gamma_{i}$ are given by

$$
\alpha_{0}=0, \quad \alpha_{1}=1, \quad \gamma_{0}=\frac{1}{2}, \quad \gamma_{1}=0, \quad \alpha_{i}=(-1)^{(i-1)} C_{2 i-2}^{i-1}, \quad \gamma_{i}=(-1)^{i} C_{2 i-2}^{i}, \quad i=2,3, \cdots
$$

Proof: Under the definition (11a), it is easy to see from $K_{0}$ that $\alpha_{0}=0, \gamma_{0}=\frac{1}{2}$. Notice the first equation in (州, we have

$$
\alpha_{k}=-2 \alpha_{k-1}+2 \gamma_{k-1}, \quad k=1,2, \cdots
$$

The identity 10

$$
\sum_{i=0}^{k}\left(a_{i} a_{k-i}+b_{i} c_{k-i}\right)=0, \quad k=1,2, \cdots,
$$

leads to the equation

$$
\gamma_{k}=\sum_{i=1}^{k-1}\left(-\gamma_{i} \gamma_{k-i}+\alpha_{i} \alpha_{k-i}\right), \quad k=1,2, \cdots .
$$

Using the equation (17), (18) and the combinational identity

$$
\frac{1}{k} C_{2 k-2}^{k-1}=\sum_{i=1}^{k-1} \frac{1}{i(k-i)} C_{2 i-2}^{i-1} C_{2 k-2 i-2}^{k-i-1}, \quad k=1,2, \cdots,
$$

we can complete the proof of Lemma 1 by induction. 
Under the definition (11) and (14) we have the expansions

$$
\begin{gathered}
J_{12}=h \sum_{i=0}^{\infty} d_{i} h^{i}=-h \partial-\frac{1}{2} h^{2} \partial^{2}-\left(\frac{1}{6} \partial^{3}+\frac{1}{2} q \partial+\frac{1}{2} q_{x}\right) h^{3}+O\left(h^{4}\right), \\
d_{4}=-\frac{1}{4 !} \partial^{4}-\frac{1}{4} \partial^{2} q+\partial g_{1}, \\
d_{i}=-\frac{1}{i} \partial^{i}-\frac{1}{2(i-2) !} \partial^{i-2} q+\frac{1}{(i-3) !} \partial^{i-3} g_{1}+\frac{1}{(i-4) !} \partial^{i-4} g_{2}, \quad i \geq 5, \\
J_{21}=h \sum_{i=0}^{\infty} e_{i} h^{i}=-h \partial+\frac{1}{2} h^{2} \partial^{2}-\left(\frac{1}{6} \partial^{3}+\frac{1}{2} q \partial\right) h^{3}+O\left(h^{4}\right), \\
e_{4}=\frac{1}{4 !} \partial^{4}+\frac{1}{4} q \partial^{2}+g_{1} \partial, \\
e_{i}=\frac{(-1)^{i}}{i} \partial^{i}+\frac{(-1)^{i-2}}{2(i-2) !} q \partial^{i-2}-\frac{(-1)^{i-3}}{(i-3) !} g_{1} \partial^{i-3}-\frac{(-1)^{i-4}}{(i-4) !} g_{2} \partial^{i-4}, \quad i \geq 5 .
\end{gathered}
$$

We define $\widetilde{J}=\left(\begin{array}{cc}0 & \widetilde{J}_{21} \\ \widetilde{J}_{12} & 0\end{array}\right)$ by requiring that

$$
J \widetilde{J}=I .
$$

Then it is found that

$$
\begin{aligned}
& \widetilde{J}_{12}=h^{-1} \sum_{i=0}^{\infty} \widetilde{d}_{i} h^{i}=-h^{-1} \partial^{-1}+\frac{1}{2}+\left(\frac{1}{2} q \partial^{-1}-\frac{1}{12} \partial\right) h+O\left(h^{2}\right), \\
& \widetilde{J}_{21}=h^{-1} \sum_{i=0}^{\infty} \widetilde{e}_{i} h^{i}=-h^{-1} \partial^{-1}-\frac{1}{2}+\left(\frac{1}{2} \partial^{-1} q-\frac{1}{12} \partial\right) h+O\left(h^{2}\right),
\end{aligned}
$$

where $\widetilde{d}_{i}, \widetilde{e}_{i}$ are determined by recurrence formulas

$$
\widetilde{d}_{k}=\partial^{-1} \sum_{i=1}^{k} d_{i} \widetilde{d}_{k-i}, \quad \widetilde{e}_{k}=\partial^{-1} \sum_{i=1}^{k} e_{i} \widetilde{e}_{k-i}
$$

Then it is found that

$$
\widetilde{J} J f=f+\eta,
$$

where function vector $\eta$ comes from the integration. The equation (21) and (23) implies that $J \eta=0$. Since the kernel of $J$ is $\left\{K_{0}, K_{1}\right\}$, we have the following lemma.

Lemma 2. The $\widetilde{J}$ defined by equations (21) and (22) satisfies

$$
J \widetilde{J}=I, \quad \widetilde{J} J f=f+\xi K_{1}+\delta K_{0},
$$

where $\xi, \delta$ are constants. 
Lemma 3. Under the definition (11a), we have the expansion

$$
\begin{gathered}
S \equiv \frac{1}{4}(\widetilde{J} G)^{2}+\widetilde{J} G=\left(\begin{array}{cc}
S_{11} & S_{12} \\
S_{21} & S_{22}
\end{array}\right), \\
S_{11}=S_{22}=\left(\frac{1}{4} \partial^{2}+\frac{1}{2} \partial^{-1} q \partial+\frac{1}{4} \partial^{-1} q_{x}\right) h^{2}+O\left(h^{3}\right), \quad S_{12}=S_{21}=\left(\frac{1}{2} \partial^{-1} q \partial+\frac{1}{4} \partial^{-1} q_{x}\right) h^{2}+O\left(h^{3}\right), \\
\quad S_{i j}+S_{k l}=B_{0}^{-1} B_{1} h^{2}+O\left(h^{3}\right),
\end{gathered}
$$

where $(i, j, k, l) \in\{(1,1,1,2),(1,1,2,1),(1,2,2,2),(2,1,2,2)\}$,

Proof: Under the definition (11a), we have the following expansions

$$
\begin{aligned}
& G_{11}=-2 h \partial-\left(\frac{1}{3} \partial^{3}+q \partial+\frac{1}{2} q_{x}\right) h^{3}+O\left(h^{4}\right), \\
& G_{12}=2 h \partial+h^{2} \partial^{2}+\left(\frac{1}{3} \partial^{3}+\frac{1}{2} q \partial+q_{x}\right) h^{3}+O\left(h^{4}\right), \\
& G_{21}=2 h \partial-h^{2} \partial^{2}+\left(\frac{1}{3} \partial^{3}+\frac{1}{2} q \partial-\frac{1}{2} q_{x}\right) h^{3}+O\left(h^{4}\right), \\
& G_{22}=-2 h \partial-\left(\frac{1}{3} \partial^{3}+2 q \partial+q_{x}\right) h^{3}+O\left(h^{4}\right) .
\end{aligned}
$$

Set

$$
T \equiv \widetilde{J} G=\left(\begin{array}{cc}
0 & \widetilde{J}_{21} \\
\widetilde{J}_{12} & 0
\end{array}\right)\left(\begin{array}{ll}
G_{11} & G_{12} \\
G_{21} & G_{22}
\end{array}\right)=\left(\begin{array}{cc}
T_{11} & T_{12} \\
T_{21} & T_{22}
\end{array}\right),
$$

then the operator has the expansion

$$
\begin{gathered}
T_{11}=-2+\frac{1}{2} h^{2} q+O\left(h^{3}\right), \quad T_{12}=2+h \partial+\left(\frac{1}{2} \partial^{2}+q\right) h^{2}+O\left(h^{3}\right), \\
T_{21}=2-h \partial+\left(\frac{1}{2} \partial^{2}-\frac{1}{2} \partial^{-1} q_{x}\right) h^{2}+O\left(h^{3}\right), \quad T_{22}=-2+\frac{1}{2} h^{2} \partial^{-1} q \partial+O\left(h^{3}\right) .
\end{gathered}
$$

Denote

$$
T^{2}=\widetilde{J} G \widetilde{J} G=\left(\begin{array}{cc}
M_{11} & M_{12} \\
M_{21} & M_{22}
\end{array}\right),
$$

then we get

$$
\begin{aligned}
& M_{11}=8+\left(\partial^{2}-\partial^{-1} q_{x}\right) h^{2}+O\left(h^{3}\right), \quad M_{12}=-8-4 h \partial+\left(-2 \partial^{2}-3 q+\partial^{-1} q \partial\right) h^{2}+O\left(h^{3}\right), \\
& M_{21}=-8+4 h \partial+\left(-2 \partial^{2}+2 q+\partial^{-1} q_{x}\right) h^{2}+O\left(h^{3}\right), \quad M_{22}=8+\left(\partial^{2}+\partial^{-1} q_{x}\right) h^{2}+O\left(h^{3}\right) .
\end{aligned}
$$

The expansions of (26) and (27) lead to Lemma 2.

Lemma 4. We have

$$
T K_{i}=\widetilde{J} G K_{i}=K_{i+1}+\delta_{i+1} K_{0}, \quad i=0,1, \cdots,
$$


where

$$
\delta_{i}=-2\left(\alpha_{i}+\gamma_{i}\right)=(-1)^{i} \frac{2}{i} C_{2 i-2}^{i-1}, \quad i=1,2, \cdots .
$$

Proof: The equations (5) and (24) give rise to

$$
T K_{i}=\widetilde{J} G K_{i}=K_{i+1}+\xi_{i+1} K_{1}+\delta_{i+1} K_{0} .
$$

It follows from (15), (17) and (26) that

$$
\xi_{i+1}=0, \quad \delta_{i+1}=-2\left(\alpha_{i+1}+\gamma_{i+1}\right),
$$

which together with (16) leads to (29).

If we define

$$
W \equiv \frac{1}{4} G \widetilde{J} G+G=\left(W_{i j}\right), \quad 1 \leq i, j \leq 2,
$$

it follows from the proof of Lemma 2 that

$$
W_{11}=W_{22}=\left(-\frac{1}{2} q \partial-\frac{1}{4} q_{x}\right) h^{3}+O\left(h^{4}\right), \quad W_{12}=W_{21}=\left(-\frac{1}{4} \partial^{3}-\frac{1}{2} q \partial-\frac{1}{4} q_{x}\right) h^{3}+O\left(h^{4}\right) .
$$

Then we arrive following Propositions.

Proposition 1. The relation between the Poisson tensors of the Toda hierarchy and those of the KdV hierarchy is as follows

$$
J=-B_{0}\left(\begin{array}{ll}
0 & 1 \\
1 & 0
\end{array}\right) h+O\left(h^{2}\right), \quad W_{i j}+W_{k l}=-B_{1} h^{3}+O\left(h^{4}\right),
$$

where $(i, j, k, l) \in\{(1,1,1,2),(1,1,2,1),(1,2,2,2),(2,1,2,2)\}$.

Proposition 2. Let

$$
w(n)=-2+\frac{1}{2} q(x) h^{2}, \quad v(n)=1+\frac{1}{2} q(x) h^{2},
$$

then

$$
\widetilde{P}_{k} \equiv \sum_{i=0}^{2 k} \beta_{k, i} K_{i}=\frac{1}{2} P_{k} h^{2 k}\left(\begin{array}{l}
1 \\
1
\end{array}\right)+O\left(h^{2 k+1}\right), \quad k \geq 1
$$

and

$$
\left(\begin{array}{c}
w \\
v
\end{array}\right)_{t_{k}}+\frac{1}{h^{2 k-1}} J \widetilde{P}_{k}=\frac{1}{2}\left(q_{t_{k}}-B_{0} P_{k}\right) h^{2}\left(\begin{array}{l}
1 \\
1
\end{array}\right)+O\left(h^{3}\right), \quad k \geq 1
$$

where

$$
\begin{gathered}
\beta_{k, 2 k}=\left(\frac{1}{4}\right)^{k-1}, \quad \beta_{k, i}=\frac{1}{4} \beta_{k-1, i-2}+\beta_{k-1, i-1}, \quad 2 \leq i \leq 2 k-1 \\
\beta_{k, 1}=\beta_{k-1,0}+\frac{1}{4} \sum_{i=0}^{2 k-2} \beta_{k-1, i} \delta_{i+1}, \quad \beta_{k, 0}=\sum_{i=0}^{2 k-2} \beta_{k-1, i}\left(\frac{1}{2} \delta_{i+1}+\frac{1}{4} \delta_{i+2}\right),
\end{gathered}
$$


with

$$
\beta_{1,0}=-2, \quad \beta_{1,1}=2, \quad \beta_{1,2}=1, \quad \text { and } \quad \delta_{i}=(-1)^{i} \frac{2}{i} C_{2 i-2}^{i-1}, \quad i=1,2, \cdots .
$$

Proof: Under the definition (31), it is easy to verify the Proposition 2 for $k=1$. By induction we have

$$
\begin{aligned}
& \left(\frac{1}{4}(\widetilde{J} G)^{2}+\widetilde{J} G\right) \widetilde{P}_{k-1}=\left(\frac{1}{4} \widetilde{J} G+1\right) \sum_{i=0}^{2 k-2} \beta_{k-1, i}\left(K_{i+1}+\delta_{i+1} K_{0}\right) \\
= & \frac{1}{4} \sum_{i=0}^{2 k-2} \beta_{k-1, i}\left(K_{i+2}+\delta_{i+2} K_{0}+\delta_{i+1} K_{1}+\delta_{i+1} \delta_{1} K_{0}\right)+\sum_{i=0}^{2 k-2} \beta_{k-1, i}\left(K_{i+1}+\delta_{i+1} K_{0}\right) \\
= & \frac{1}{4} \beta_{k-1,2 k-2} k_{2 k}+\sum_{i=2}^{2 k-1}\left(\frac{1}{4} \beta_{k-1, i-2}+\beta_{k-1, i-1}\right) K_{i}+\left(\beta_{k-1,0}+\frac{1}{4} \sum_{i=0}^{2 k-2} \beta_{k-1, i} \delta_{i+1}\right) K_{1} \\
& +\sum_{i=0}^{2 k-2} \beta_{k-1, i}\left(\frac{1}{2} \delta_{i+1}+\frac{1}{4} \delta_{i+2}\right) K_{0}=\widetilde{P}_{k} \equiv \sum_{i=0}^{2 k} \beta_{k, i} K_{i} .
\end{aligned}
$$

Using $S$ in (25), the following approximation is deduced

$$
\begin{aligned}
& \left(\frac{1}{4}(\widetilde{J} G)^{2}+\widetilde{J} G\right) \widetilde{P}_{k-1}=\left(\begin{array}{ll}
S_{11} & S_{12} \\
S_{21} & S_{22}
\end{array}\right)\left(\frac{1}{2} P_{k-1} h^{2 k-2}\left(\begin{array}{l}
1 \\
1
\end{array}\right)+O\left(h^{2 k-1}\right)\right) \\
= & \left(\begin{array}{c}
S_{11}+S_{12} \\
S_{21}+S_{22}
\end{array}\right)\left(\frac{1}{2} P_{k-1} h^{2 k-2}+O\left(h^{2 k-1}\right)\right) \\
= & \frac{1}{2} B_{0}^{-1} B_{1} P_{k-1} h^{2 k}\left(\begin{array}{l}
1 \\
1
\end{array}\right)+O\left(h^{2 k+1}\right)=\frac{1}{2} P_{k} h^{2 k}\left(\begin{array}{l}
1 \\
1
\end{array}\right)+O\left(h^{2 k+1}\right),
\end{aligned}
$$

which together with the equation (34) gives rise to the equation (32). The equation (33) can be obtained by combining the equation (30), (31) and (32).

For example, the first three $\widetilde{P}_{k}$ 's are

$$
\begin{aligned}
& \widetilde{P}_{1}=-2 K_{0}+2 K_{1}+K_{2}=\left(\begin{array}{c}
2+w \\
1-\frac{1}{v}
\end{array}\right), \\
& \widetilde{P}_{2}=\frac{3}{2} K_{0}-K_{1}+\frac{3}{2} K_{2}+\frac{3}{2} K_{3}+\frac{1}{4} K_{4}, \\
& \widetilde{P}_{3}=-\frac{5}{4} K_{0}+\frac{3}{4} K_{1}-\frac{5}{8} K_{2}+\frac{5}{4} K_{3}+\frac{15}{8} K_{4}+\frac{5}{8} K_{5}+\frac{1}{16} K_{6} .
\end{aligned}
$$

Proposition 3. Let

$$
w(n)=-2+\frac{1}{2} q(x) h^{2}+\frac{1}{8} q_{x}(x) h^{3}, \quad v(n)=1+\frac{1}{2} q(x) h^{2}-\frac{1}{8} q_{x}(x) h^{3},
$$

then we have

$$
Q_{k} \equiv\left(\begin{array}{c}
Q_{k, 1} \\
Q_{k, 2}
\end{array}\right) \equiv \sum_{i=0}^{2 k-1} \widetilde{\beta}_{k, i} K_{i}
$$




$$
Q_{k, 1}+Q_{k, 2}=P_{k} h^{2 k}+O\left(h^{2 k+1}\right), \quad k \geq 2,
$$

and

$$
\left(w_{t_{k}}+v_{t_{k}}\right)+\frac{1}{h^{2 k-1}}\left(J_{12} Q_{k, 2}+J_{21} Q_{k, 1}\right)=\left(q_{t_{k}}-B_{0} P_{k}\right) h^{2}+O\left(h^{3}\right), \quad k \geq 2
$$

where

$$
\begin{gathered}
\widetilde{\beta}_{k, 2 k-1}=\left(\frac{1}{4}\right)^{k-2}, \quad \widetilde{\beta}_{k, i}=\frac{1}{4} \widetilde{\beta}_{k-1, i-2}+\widetilde{\beta}_{k-1, i-1}, \quad 2 \leq i \leq 2 k-2 \\
\widetilde{\beta}_{k, 1}=\widetilde{\beta}_{k-1,0}+\frac{1}{4} \sum_{i=0}^{2 k-3} \widetilde{\beta}_{k-1, i} \delta_{i+1}, \quad \widetilde{\beta}_{k, 0}=\sum_{i=0}^{2 k-3} \widetilde{\beta}_{k-1, i}\left(\frac{1}{2} \delta_{i+1}+\frac{1}{4} \delta_{i+2}\right),
\end{gathered}
$$

with $\delta_{i}$ are given in Proposition 2, and

$$
\widetilde{\beta}_{2,0}=4, \quad \widetilde{\beta}_{2,1}=-2, \quad \widetilde{\beta}_{2,2}=2, \quad \widetilde{\beta}_{2,3}=1 .
$$

Proof: It is also easy to check Proposition 3 for $k=2$. Apply the operator $S$ on $Q_{k-1}$, we can get the similar equation as (34) ( $\widetilde{P}_{k-1}$ is substituted by $Q_{k-1}$ and the superscript of summation is $2 k-3$ and $2 k-2$ instead of $2 k-2$ and $2 k-1$, respectively). Upon the assumption of Proposition 3 for $k-1$

$$
Q_{k-1,1}+Q_{k-1,2}=P_{k-1} h^{2 k-2}+O\left(h^{2 k-1}\right)
$$

we have

$$
\begin{aligned}
& Q_{k, 1}+Q_{k, 2}=\left(S_{11}+S_{21}\right) Q_{k-1,1}+\left(S_{12}+S_{22}\right) Q_{k-1,2} \\
& =B_{0}^{-1} B_{1} P_{k-1} h^{2 k}+O\left(h^{2 k+1}\right)=P_{k} h^{2 k}+O\left(h^{2 k+1}\right) .
\end{aligned}
$$

The above formulation and (30) lead to (39).

The first two $Q_{k}$ 's read

$$
Q_{2}=4 K_{0}-2 K_{1}+2 K_{2}+K_{3}, \quad Q_{3}=-3 K_{0}+\frac{3}{2} K_{1}-K_{2}+\frac{3}{2} K_{3}+\frac{3}{2} K_{4}+\frac{1}{4} K_{5} .
$$

Proposition 4. Let

$$
w(n)=-2+\frac{1}{2} q(x) h^{2}+\frac{1}{8} q_{x}(x) h^{3}-\frac{1}{32} q^{2}(x) h^{4}, \quad v(n)=1+\frac{1}{2} q(x) h^{2}-\frac{1}{8} q_{x}(x) h^{3}+\frac{1}{32} q^{2}(x) h^{4},
$$

then

$$
R_{k} \equiv \sum_{i=0}^{2 k-1} \widetilde{\beta}_{k, i} K_{i}=\frac{1}{2} P_{k} h^{2 k}\left(\begin{array}{l}
1 \\
1
\end{array}\right)+O\left(h^{2 k+1}\right), \quad k \geq 2
$$

and

$$
\left(\begin{array}{c}
w \\
v
\end{array}\right)_{t_{k}}+\frac{1}{h^{2 k-1}} J R_{k}=\frac{1}{2}\left(q_{t_{k}}-B_{0} P_{k}\right) h^{2}\left(\begin{array}{l}
1 \\
1
\end{array}\right)+O\left(h^{3}\right), \quad k \geq 2 .
$$

where $\widetilde{\beta}_{k, i}$ are defined in Proposition 3 .

Proposition 4 can be proved by the similar method used in the proof of Proposition 2. 
Remark 1. The $g(x)$ in (11a) is assumed to be a polynomial of $q(x)$ and the derivatives of $q$ and is chosen in such way in [5] that when $q(x)$ is a solution of $\mathrm{KdV}$ flows, the $w(n), v(n)$ are approximate solution to the Toda flows to high accuracy. The geometric meaning of this operation is not clear. Comparing the above propositions, we can conclude that the higher accuracy is introduced in the definition (11a), the fewer components are needed in the recombination method to recover the KdV hierarchy through the limit process. For example, under (11) with (14a), we need combination of $K_{2}, \cdots, K_{6}$ to have $\mathrm{KdV}$ equation (10) as a continuous limit, however, for (11) with (14b), the combination of $K_{2}, \cdots, K_{5}$ goes to $\mathrm{KdV}$ equation (10) in continuous limit.

Remark 2. The proposition 1 implies that the Poisson structure of KdV hierarchy is recovered combining the entries of the matrices of the Toda Poisson tensors in continuous limit. However the geometric meaning of such combinations of different entries is not clear.

In addition, apart from the commuting vector fields, we can also show that the conserved functionals, the Lax pairs and restricted flows for Toda hierarchy go to those for KdV hierarchy in continuous limits.

\section{Acknowledgment}

The work was supported by the National Basic Research Project for Nonlinear Sciences and the Doctorate Dissertation Foundation of Tsinghua University. We are grateful to the anonymous referee for the helpful comments.

\section{References}

[1] M. Toda and M. Wadati, J. Phys. Soc. Japan, 34 (1973) 18.

[2] K.M. Case, J. Math. Phys., 14 (1974) 916; K.M. Case and M. Kac, J. Math. Phys., 14 (1974) 594.

[3] B.A. Kupershmidt, Discrete Lax equations and differential-difference calculus (Astérisque 123, 1985, Soc. Math. de France).

[4] Yunbo Zeng and S. Rauch-Wojciechowski, J. Phys. A: Math. Gen., 28 (1995) 3825.

[5] D. Gieseker, Commun. Math. Phys., 181 (1996) 587.

[6] C. Morosi and L. Pizzocchero, Commun. Math. Phys., 180 (1996) 505.

[7] Yunbo Zeng, Acta Mathematicae Applicatae Sinica, 14 (1998) 176.

[8] C. Morosi and L. Pizzocchero, J. Phys. A: Math. Gen., 31 (1998) 2727. 
[9] C. Morosi and L. Pizzocchero, Revews in Math. Phys., 10 (1998) 235.

[10] Guizhang Tu, J. Phys. A, 23 (1990) 3903.

[11] A.C. Newell, Soliton in mathematics and physics (SIAM, Philadelphia, 1985). 